\title{
Empirical forecasting of HF-Radar velocity using
}

\section{Genetic Algorithms}

\author{
Alejandro Orfila, Anne Molcard, Juan M. Sayol, Julien Marmain, Lucio Bellomo, \\ Celine Quentin and Yves Barbin
}

\begin{abstract}
We present a coastal ocean current forecasting system using exclusively past observations of a High Frequency Radar (HF-Radar). The forecast is made by developing a new approach based on physical and mathematical results of nonlinear dynamical systems theory that allows to obtain a predictive equation for the currents. Using radial velocities from two HF-Radar stations, the spatio-temporal variability of the fields is first decomposed using the Empirical Orthogonal Functions. The amplitudes of the most relevant modes representing their temporal evolution are then approximated with functions obtained through a Genetic Algorithm. These functions will be then combined to obtain the hourly currents at the area for the next 36 hours. The results indicate that after 4 hours and for an
\end{abstract}

Manuscript submitted on March 18, 2014. Authors would like to thank financial support from TOSCA MED Project (G-MED09-425), from MEDESS-4MS MED Project (2S-MED11-01) and from MICINN Project (CGL2011-22964). J.M.Sayol is supported by the PhD CSIC-JAE program co-funded by the European Social Fund (ESF) and CSIC. This work was partially performed while A. Orfila was a visiting scientist at the Mediterranean Institute of Oceanography at Universite de Toulon-Sud through a MECD fellowship (PRX12/00454). Comments from two anonymous reviewers are greatly acknowledged

A. Orfila and J.M. Sayol are with the Department of Marine Technologies and Operational Oceanography, IMEDEA (CSIC-UIB), Balearic Islands, 07190, Spain (e-mail: aorfila@imedea.uib-csic.es; jsayol@imedea.uib-csic.es)

A. Molcard, J. Marmain, L. Bellomo, C. Quentin and Y. Barbin are with Mediterranean Institute of Oceanography. Université de ToulonVar,Aix-Marseille Université, CNRS, IRD, MIO UM 110, 83957 La Garde, France (e-mail: molcard@univ-tln.fr; marmain@univ-tln.fr; bellomo@univ-tln.fr; quentin@univ-tln.fr; yves.barbin@univ-tln.fr) 
horizon of 24 hours, the computed predictions provide more accurate current fields than the latest available field (i.e. persistent field).

\section{Index Terms}

Operational Oceanography, HF-Radar, Empirical modelling

\section{INTRODUCTION}

NOWLEDGE of coastal currents either for forecasting or nowcasting purposes is a relevant
scientific and technological issue that has been receiving increasing attention in the last decades due to the large importance that shelves have in the economy, the biogeochemical cycles or in the engineering activities. The preservation of the water quality and the conservation of coastal areas are two important objectives of developed societies essential to guarantee the sustainable management of coasts. By contrast, the degradation of shallow waters associated with accidental or illegal spills is directly related with the losses of habitats being among the largest threats in marine environments [16], [28].

Coasts are the transition areas between open ocean and land. The hydrodynamic in these areas is the result of a complex interaction of processes where the energetic inputs from the atmosphere (mainly through wind and heat fluxes) and those from the ocean boundaries (density gradients and energy provided by waves) balance with dissipation by coast and the bottom boundary layer and with the input of mass through rivers run-off. Besides, the complexity of the dynamics of coastal seas is increased by the nonlinear interactions among the physical processes that sometimes generate well defined locally intense currents. Therefore, coastal areas have specific dynamical features with different spatial and temporal resolution from open ocean that need to be constantly monitored in order to have an accurate representation of the physical mechanisms driving the dynamics.

The forecasting of coastal currents constitutes one of the most important challenges in geosciences. This relevance is given by the important role that coasts play in human related activities. Maritime traffic, search and rescue operations, environmental control, military operations, etc, are some examples of the 
activities that require accurate and continuous forecast of currents in coastal seas. Unfortunately, the strong space-time variability of these areas together with the complexity of the processes inherent there make the prediction a difficult task. Although numerical modelling is the most common approach to forecast the ocean it requires continuous data support, which sometimes is hardly achieved. On the other hand, numerical model performance is quickly degraded if only surface data is provided. Besides the numerical modelling approach, any dynamical system like the ocean can be empirically modelled by expressing time evolving measurements in a suitable functional form.

As a consequence of this importance, the establishment of coastal observing systems has been recently identified as an important component of the marine strategy by the European Commission (2010-2013) as well as for most advanced countries with economically significant coastal areas [34]. In this respect, a significant effort has been devoted in the last years to different initiatives worldwide regarding Coastal Observing Systems (COS). These observatories, such as Integrated Marine Observing System-IMOS in Australia, the Ocean Observatories Initiative-OOI and the different regional components from IOOS (Integrated Ocean Observing System) in USA, Neptune and Venus in Canada, Cosyna in Germany, SOCIB (Balearic Islands Coastal Observing \& Forecasting System) in Spain, MOOSE (Mediterranean Ocean Observing System for the Environment) in France among others, routinely acquire coastal ocean variables presenting them for the end user in a readable format providing new scientific insights about the dynamics of coastal seas.

The advent of new remote sensing data and techniques has yielded a completely new perspective of the observation of the oceans and coasts. As a result, the historical lack of data in the ocean is now being partly filled by regional and global synoptic views of the spatial structure of the ocean. Among this type of platforms appears the HF-Radar as one of the key components of COS for observing coastal currents.

HF-Radar is a monitoring system that determines the ocean surface currents indirectly through electromagnetic waves emitted from a set of antennas and Bragg backscattered from the sea surface. The radial component of the current is estimated by measuring the Doppler shift at the surface layer [9]. The 
measured value is a vertical average of the actual current component through an exponential weighting function with a characteristic depth of the order of $1 \mathrm{~m}$ and depending on the HF-Radar working frequency [31]. Today, more than 450 of these platforms are operating around the globe [10].

Nevertheless COS provide only the observed surface velocities for the present state of the ocean and to infer the future state for operational purposes one should implement additional processes. Traditionally, the forecasting of coastal currents is made by using numerical models that integrate the primitive NavierStokes equations forward in time. Numerical models are not absent of problems mainly related with the turbulence parametrization and the initialization of ocean fields and usually, they poorly describe coastal dynamics both in terms of resolution and processes resolved. Coastal ocean model forecasts typically rely on data assimilation to partly compensate for these uncertainties.

Since the work of [23], who developed methods for exploring the limits of predictability in the atmosphere, a new perspective based on the ideas of nonlinear dynamics and chaos emerged and has been applied to many different disciplines. Currently, methods for the prediction of these type of systems based on observations exist in almost all geophysical fields including classical fluid dynamics, physical oceanography, meteorology, astrophysics, hydromagnetics, etc (e.g., [32], [35], [36]). In general those approaches obtain a proxy of the dynamical system by model fitting the observed phenomena.

Under this point of view, some recent works used surface currents from HF-Radar to develop semiempirical models based on statistical approaches from past observations in order to obtain operational forecasting of currents for oil spill dispersion or search and rescue operations [10], [18].

Motivated by such approach, we present a system for the short term operational forecasting of surface currents using an evolutionary algorithm. The main differences from previous works are that first, we construct the dynamical system directly from radar radial velocities and second, no information is required from the atmospheric forcing. The manuscript first presents the HF-Radar data used in this development as well as the methods implemented including the DINEOF (Data Interpolating Empirical Orthogonal Functions) used to fill the gaps, the spatial and temporal decomposition of current variability using EOF 


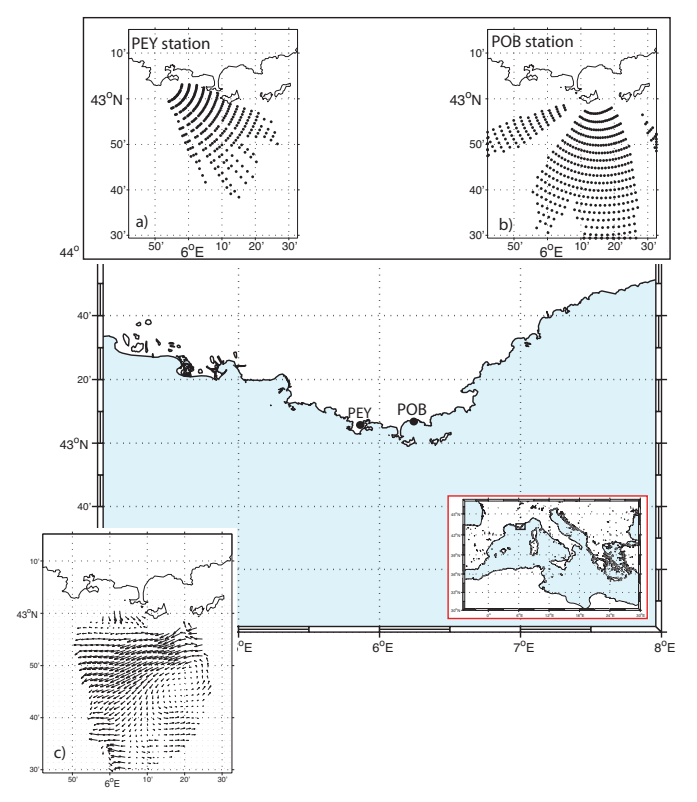

Fig. 1. Geographical location of the Study Area. The box indicates the location of the HF-Radar facility. On the the top panel it is displayed the location of the PEY (a) and POB (b) antennas as well as the spatial points where radial velocities are given. A snapshot of the reconstructed Cartesian $u-v$ velocities is displayed on (c) for June $20^{\text {th }} 2013$.

(Empirical Orthogonal Functions) and the SSA (Singular Spectrum Analysis) filtering. This Section ends with the evolutionary algorithm based on the Darwinian theories of natural selection and survival. In Section 3 the main results are presented and physically discussed and, finally, we conclude the work providing some ideas for the development of the fully operative operational system.

\section{DATA AND Methods}

\section{A. HF-Radar data in the Var coast}

Surface currents are measured hourly by HF-Radar along the Var coastal area (Provence-Alpes-Cotes d'Azur PACA region in the south east of France) since May 2010 (Fig. 1).

The dynamics in this coastal area have still not been studied and observed as widely as the nearby Gulf of Lions and Ligurian Sea, even given its strategic position regarding the northwestern Mediterranean coastal current (Northern Current, NC hereafter) pathway. This coastal region is characterized by a steep topography (1000 m depth reached within several $\mathrm{km}$ ), a complex coastline and the presence of numerous islands. The incoming westward NC interacts with this rough topography and is subject to very strong 
wind episodes (Mistral), leading to possible instabilities of the coastal current, and the generation of meanders [19]. The wealth of this dynamics led to the installation of a radar system in order to monitor the variability of the coastal current, just upstream of the Gulf of Lions.

The HF-Radar is a WERA (WEllen RAdar) [20] system consisting of two sites operating at $16.15 \mathrm{MHz}$ with a bandwidth of $50 \mathrm{kHz}$.

The first location, Fort Peyras -PEY- (Fig. 1(a)) has a quasi-monostatic configuration with an irregular, W-shaped, 8-antenna receiving array and a single emitting antenna. The peculiarity of the receiving array geometry is imposed by the environment of the site. The second site -POB- is a system with a bistatic configuration, with the emitter at Porquerolles Island located $17 \mathrm{~km}$ far from the receiver sited in Cap Bénat, a regular linear 8-antenna array (Fig.1(b)). The radial current maps are produced on a grid with azimuthal step of $\pi / 45 \mathrm{rad}$ and nominal range resolution of $3 \mathrm{~km}$. Here the radial velocities are by convention considered as positive when directed towards the antennas and as negative when directed away from them (see Fig. 2). Zero radial velocities indicate that only the perpendicular (azimuthal) velocity component may be important at the specific point.

The integration is performed over roughly 1 hour and the method used to determine current velocities is a Direction Finding method inspired by the MUSIC (MUltiple SIgnal Characterization) algorithm [1], [22], [29] which is routinely used at MIO in its HF-Radar installations [2], [24]. Cartesian velocities are reconstructed on a regular $2 \mathrm{~km} \times 2 \mathrm{~km}$ grid from the two radial maps through an unweighted least squares method applied locally within a circle of $3 \mathrm{~km}$ radius around each grid point [21], [30]. Although the nominal radial current velocity resolution is $\approx 1.8 \mathrm{~cm} / \mathrm{s}$, a higher value should be retained because of the effect of FFT (Fast Fourier Transform) windowing in the signal processing chain (bounded at twice the nominal one). The regular grid step is rather arbitrarily set at $2 \mathrm{~km}$ and the GDOP (Geometric Dilution of Precision) results in a global Cartesian velocity accuracy of $\pm 2.5 \mathrm{~cm} / \mathrm{s}$ in the worst case (the most southern points). The coverage of the system is between $5^{\circ} 50^{\prime}-6^{\circ} 25^{\prime} \mathrm{E}$ and $42^{\circ} 30^{\prime}-43^{\circ} 01^{\prime} \mathrm{N}$ (Fig. 1 (c)). The surface current snapshot resulting from the 2 radial maps shown for the $20^{\text {th }}$ of June 2013, 
clearly displays the NC slightly bending south eastward.

\section{B. Filling spatial gaps: DINEOFs}

Since some radial cells may be missing during singular measurements - either because of the intrinsically discrete nature of the inversion algorithm or because of outliers that are eliminated in the first stages of data processing - the final Cartesian data might present gaps. To fill these gaps in the radial velocity fields, the DINEOF technique is used [13]. DINEOF has been already applied to reconstruct the SST of satellite images in the Adriatic Sea [7] and in a multi-variate approach to reconstruct missing data in SST, Chl-a concentration and QuickSCAT winds [6]. DINEOF supplies missing values in a specific observation from an optimal number of EOF determined by a cross-validation technique [14] and with a temporal filtering of covariance matrices to improve the interpolation process. The advantage of using DINEOF compared to more traditional optimal interpolation techniques to reconstruct data fields is that DINEOF fills gaps using only available data, so there is no subjectivity in parameter estimation [8]. The DINEOF was applied to the the hourly radial velocities.

In this work, two different periods have been used for the forecasting: from January $29^{\text {th }}$ to April $30^{\text {th }} 2013$ and from June $1^{\text {st }}$ to August $31^{\text {st }}$ 2013. Data consisted in hourly radial velocities over 813 locations for PEY station and over 1386 locations for the POB station. Cartesian velocities are eventually reconstructed over 527 equally spaced points.

\section{Encoding data: EOF decomposition}

Strictly speaking, forecasting the HF-Radar surface velocities implies obtaining a predictive model for each time series of velocities. Since this approach is unrealistic, encoding the spatio-temporal information is needed. Here, for this purpose, we use an EOF decomposition [27].

In short, given $n_{x} \mathrm{x} n_{y}$ velocities at $n_{t}$ times, the spatio temporal velocities can be decomposed as:

$$
V_{j}(x, y, t)=\sum_{i=1}^{n_{t}} a_{i j}(t) E O F_{i j}(x, y),
$$


where $\mathrm{EOF}_{i j}$ represents the spatial pattern, $a_{i j}$ is the temporal amplitude (or weight) of each $\mathrm{EOF}_{i j}$, and $j=1,2$ the radial site to which belongs the current field. Hereinafter, the spatial and temporal patterns of the EOF-decomposition will be called modes and amplitudes, respectively. The decomposition is done by diagonalizing the reduced spatial dimension covariance matrix [17], [38]. Often, the first modes (those explaining most of the variance) can be related to a specific physical process [4], [25].

\section{Genetic programming}

The forecast approach adopted in this work relies on a genetic algorithm (using only past observations) to identify mathematical expressions that best forecast the evolution of the amplitudes associated with statistically significant EOF modes.

Explicitly, the work of Takens [33], provides the theoretical background to obtain the necessary information about the evolution of a non-linear system directly from the time series. Takens' theorem is a delay embedding theorem that states that using a sampled observable, $\left\{x_{i}\right\}=\{x(i \tau), i=1, \cdots, M\}$, where $\tau$ is the sampling time, there exists a map $P(\cdot)$ satisfying,

$$
x_{m}=P\left(x_{m-1}, x_{m-2}, \cdots, x_{m-d}\right) .
$$

Here, $d$ is the embedding dimension.

In summary, the task to build a predictive model directly from data is simplified in two steps:

- The state space reconstruction in order to use the immediate history of the timeseries to reconstruct the current state of the system, at least to a level of accuracy permitted by noise and,

- The nonlinear approximation of the dynamics of the function $P(\cdot)$ in Eq. 2, that maps the current state to a future state.

In the present work, the dynamical characterization is done in the time delay representation of the system by describing and mapping it using a genetic algorithm (GA) [3]. This approach has been successfully used to forecast nonlinear dynamical systems approximating the predictive model $P(\cdot)$ in Eq. (2) [5], [12], [15], [26]. 
The GA considers first a set of candidate equations (the initial population) of potential solutions that are subjected to evolutionary genetic steps, e.g. reproduction, crossover and mutation, selecting from the initial population those equations (individuals) that best fit the data. The strongest strings choose a mate for reproduction whereas the weakest strings become extinct. The newly generated population is subjected to mutations that change fractions of information. The evolutionary steps are repeated with the new generation ending after a number of generations -a priori- determined by the user.

The initial population is generated by random combination of the explanatory variable (the amplitudes) $a_{i}=a(i t), i=1, \cdots, N$ and randomly chosen constants combined by the four basic operators $(+,-, \times, \div)$. For a $\mu$-ahead step prediction with embedding dimension $d$, the fitness $\Delta_{j}^{2}$ of the $j$-candidate map $P_{j}$ is defined as,

$$
\Delta_{j}^{2}=\sum_{m=\mu+d}^{M}\left[x_{m}-P_{j}\left(a_{m-\mu}, \cdots a_{m-\mu-d+1}\right)\right]^{2},
$$

being $x_{m}$ and $a_{m}$ the explained and explanatory variables respectively at time $t_{m}=m t$. This fitness determines the strength of each individual to be used in the next evolutionary step. Finally, the validation of the candidate equation is carried out using data not included in the training process.

The GA was configured in such a way that $d=120$ for the embedding dimension and 1 for the time lag. The maximum number of characters allowed for each tentative equation is 20 . These parameters have been chosen after a trial error process and the same combination of parameters has been taken for all the amplitudes. Every generation consists of a population of 120 randomly generated equations with a number of 10000 generations involving $1.2 \cdot 10^{6}$ equations for each one. The CPU time used for each amplitude run was below 120 seconds.

\section{E. Filtering}

The genetic algorithm used here is particularly well-suited for forecasting nonlinear time series like those expected from ocean surface velocities. However, a filtering is required prior to the application of the GA since the variability of the observed time series results from the deterministic part of the signal 
and a random component that will be unpredictable. This "noisy" part of the signal would lead to a bad performance of the mapping $P(\cdot)$ since the GA will try to find a dynamical law for a random effect.

Filtering of the EOF amplitudes has been performed using the SSA analysis [37]. The noise-free amplitude is reconstructed by adding SSA components above a certain noise level determined by the forecasting skill of the GA, i.e., if the performance of the mapping equation provided by the GA in a training set is higher than $65 \%$ of the variance explained for the specific combination of SSA modes the signal is considered as deterministic and the noise free EOF-amplitude refined by adding additional SSA eigenvalues (one at a time) until the forecast skill begins to degrade. This method allows to train the algorithm with the deterministic part of the signal removing the unpredictable part that would produce a decreasing performance in the searching method. The percent variance explained by the retained SSA modes and the forecasting skill of the GA over the reconstructed signals are shown in Table I.

\section{RESULTS AND DISCUSSION}

The forecasting system is tested in two periods. Possible gaps on the radial velocities fields are filled using the DINEOFs. From the new complete datasets the EOFs were computed at each antenna from the hourly radial velocities measured at January $29^{\text {th }}$ to April $28^{\text {th }} 2013$ (the winter period) and from June $1^{\text {st }}$ to August $29^{\text {th }} 2013$ (the summer period). The last 36 hours (the forecast) were not used for the EOF decomposition. The amplitudes of the first four most relevant EOF modes were filtered using SSA and then a GA trained over each of the filtered amplitudes and using the best equation provided by the GA a forecast made for a 36 hours horizon by combining again the forecast of the first four amplitudes multiplied by their EOF modes and including the mean field.

The first four EOFs from the decomposition of the radial velocities have been selected for the forecasting process based on the percentage of the explained variance (a total of $67 \%$ and $70 \%$ for PEY for the first and second period respectively and of $60 \%$ and $67 \%$ for POB station for the first and second period respectively). Mean radial velocities were subtracted before the EOF analysis. Fig. 2 shows the mean radial velocity at both stations for the period corresponding to June $1^{\text {st }}$ to August $29^{\text {th }} 2013$ where 
the most remarkable signature as seen in these images is the $\mathrm{NC}$ with absolute maximum of the radial velocity around $0.3 \mathrm{~m} / \mathrm{s}$. The corresponding mean for the winter period presents a similar spatial pattern (not shown).
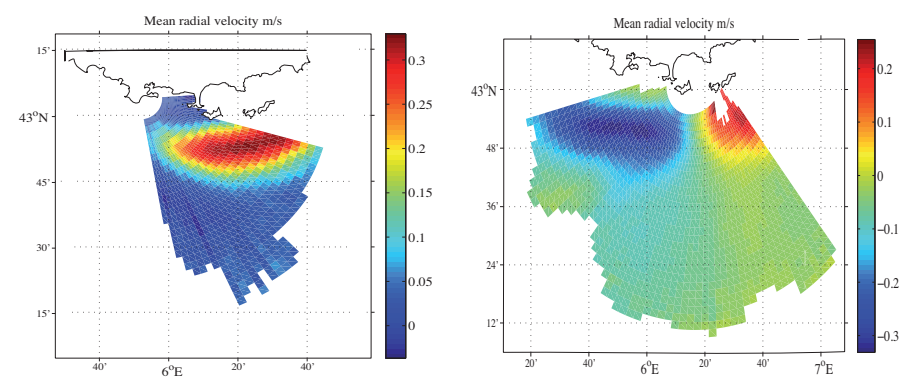

Fig. 2. Mean radial velocity in $(\mathrm{m} / \mathrm{s})$ for the PEY antennas (left) and for the POB antennas (right) for the period of June $1^{\text {st }}$ to August $29^{\text {th }} 2013$.

The first four spatial EOF modes (weighted by the maximum value of their respective amplitudes) for PEY station are displayed in Fig. 3 (a)-(d) for January 29 2013 to April $28^{\text {th }} 2013$ and in (i)-(l) for June $1^{\text {st }} 2013$ to August $29^{\text {th }} 2013$. The corresponding amplitudes of the modes are shown in Fig. 3 (e)-(h) and (m)-(p) respectively.

The first mode, which accounts $45 \%$ and $52.2 \%$ of the total variance of winter and summer periods respectively, represents the displacement of the NC towards the coast (Fig. 3 (a) and Fig. 3 (i)). While the amplitude of the first mode corresponding to the summer period (Fig. 3 (m)) has a significant component of its spectral energy between 100 and 250 hours, the amplitude of the first mode corresponding to the winter period (Fig. 3 (e)) contains the major part of the spectral energy at shorter periods $(<30$ hours) indicating the different dynamics of the ocean currents of the two situations analysed. The successive EOFs account for the first period 10\%,7\% and 5\% (Fig. 3 (b)-(d)) and for the second period 7\%, $6 \%$ and $5 \%$ (Fig. 3 (i)-(1)) respectively, and the features that appear may be interpreted as a coastal front, shear or intensification/weakening of the NC. The spectral analysis of their amplitudes highlights for the summer data (Fig. $3(\mathrm{~m})-(\mathrm{p}))$ specific signatures around the inertial and daily periods (17 hours and 24 hours) but no evident signals have been identified for the data corresponding to the winter period. 

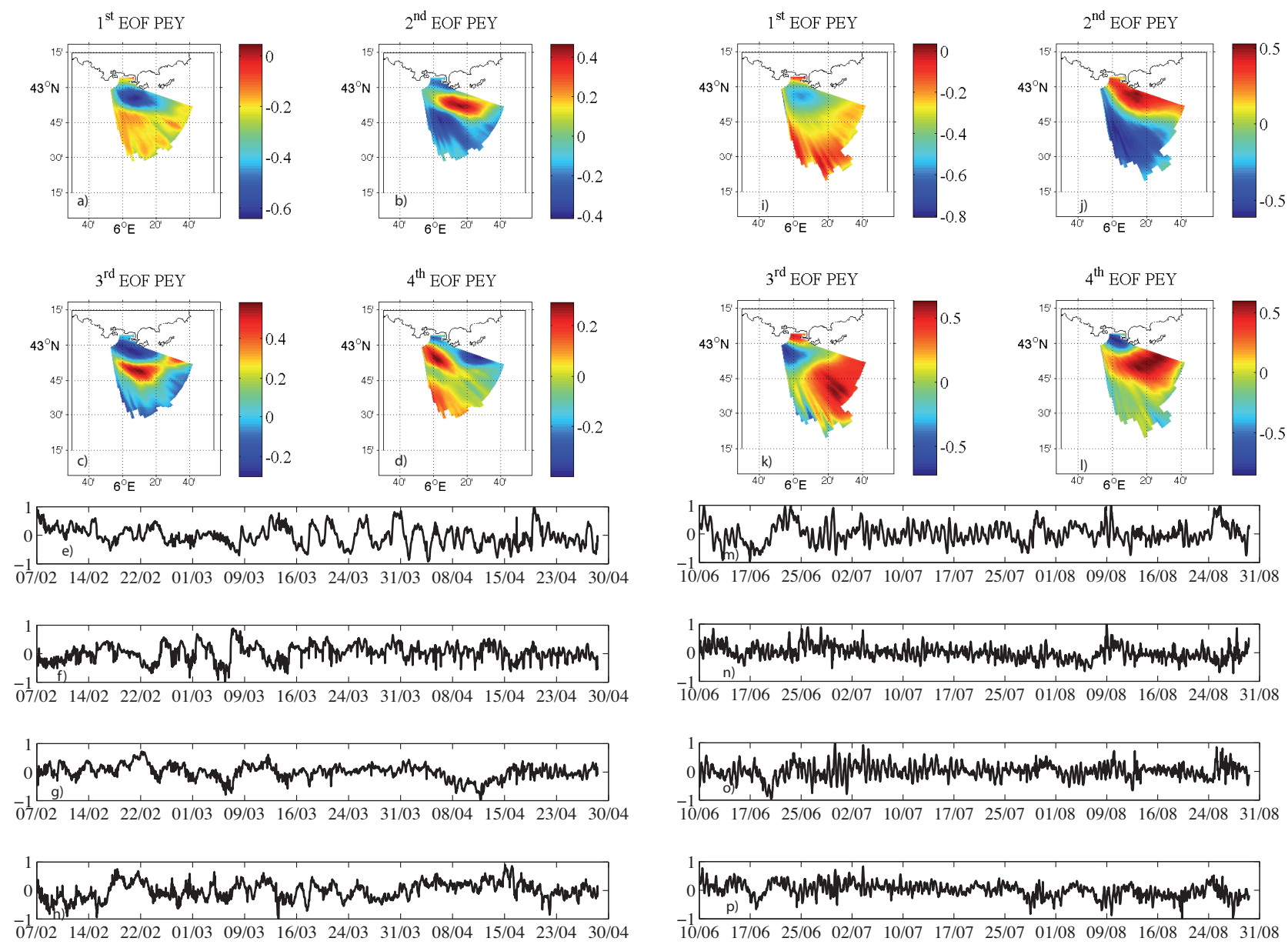

Fig. 3. PEY station. First (a), second (b), third (c) and fourth (d) EOF modes for the period of $29^{\text {th }}$ January 2013 to $28^{\text {th }}$ April 2013 and their corresponding amplitudes amplitudes in (e)-(h) respectively. The first fourth EOF modes for the period $1^{\text {st }}$ June 2013 to $29^{\text {th }}$ August 2013 in (i)-(l) and their amplitudes in (m)-(p). Units in m/s.

Regarding POB station, the first four modes for the period January $29^{\text {th }}$ to April $28^{\text {th }} 2013$ account $34 \%, 15 \%, 6 \%$ and $5 \%$ of the total variability respectively (Fig. 4 (a)-(d)) and for the period of June $1^{\text {st }}$ to August $29^{\text {th }} 201341 \%, 14 \%, 7 \%$ and 5\% respectively (Fig. 4 (i)-(l)). While the second EOF for both periods represents the amplification/weakening of the coastal current (Fig. 4 (b) and Fig. 4 (j)) the others depict its modulation in terms of width and shape. The main feature identified from their temporal amplitudes for the summer (Fig. 4 (m)-(p)) are the spectral component at 24 hours present in all the modes and the peak of energy at the spectra localized around the period of the inertial oscillations (i.e. $\sim 17$ hours) for both the second and third modes (Fig. 4 (n) and (o)). 

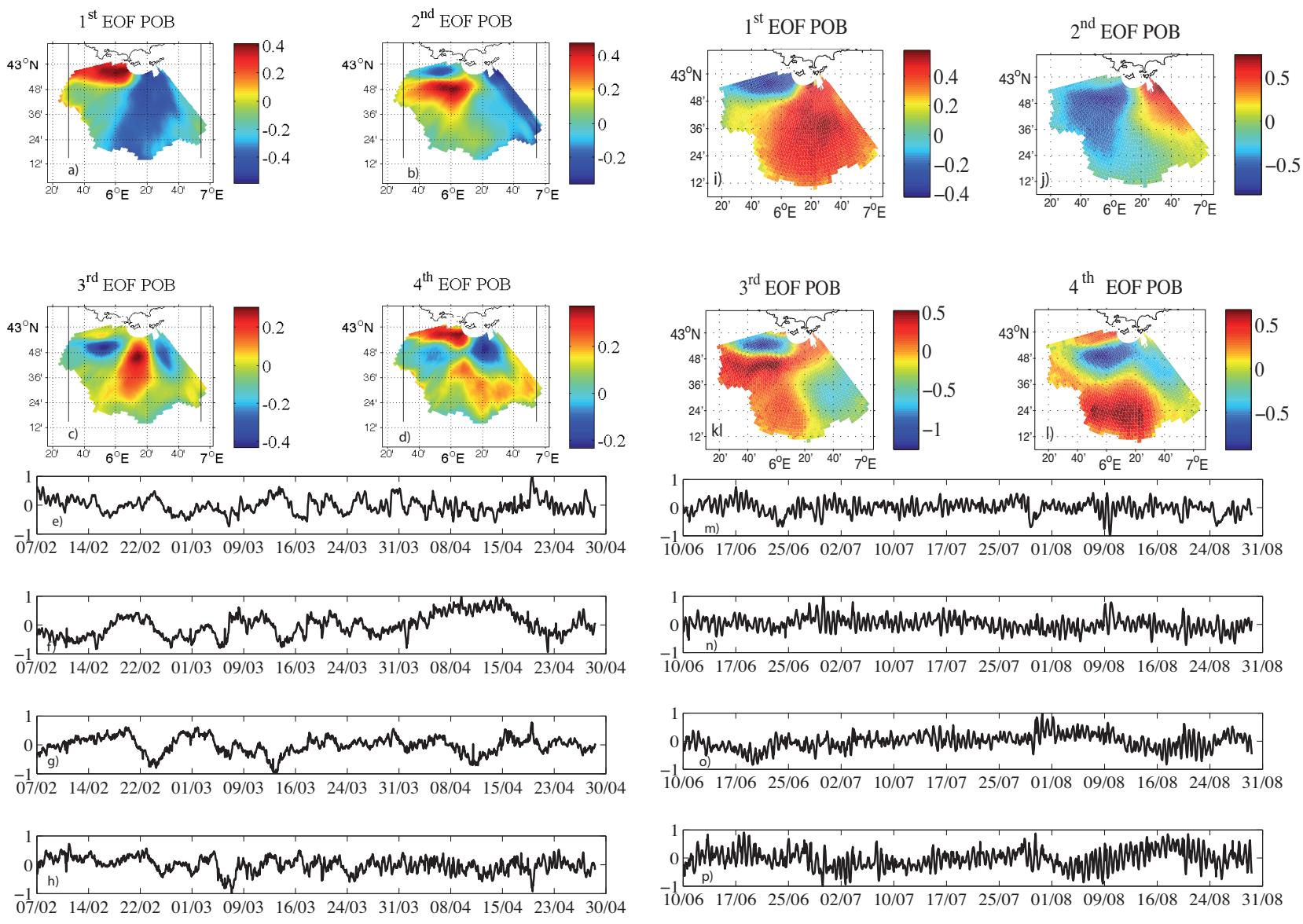

Fig. 4. POB station. First (a), second (b), third (c) and fourth (d) EOF modes for the period of $29^{\text {th }}$ January 2013 to $28^{\text {th }}$ April 2013 and their corresponding amplitudes in (e)-(h) respectively. The first fourth EOF modes for the period $1^{\text {st }}$ June 2013 to $29^{\text {th }}$ August 2013 in (i)-(1) and their amplitudes in (m)-(p). Units in $\mathrm{m} / \mathrm{s}$.

Table I shows the percentage of explained variance obtained by the best fitted equation in the validation set. The best individuals obtained by the GA for the two first amplitudes of each mode provide in general better skill than the last two indicating that some "noise" may be present in the higher mode EOF amplitudes. In fact, the inclusion of new EOFs in the forecasting process deteriorates the skill.

The forecast of the HF-radar data is made by reassembling the EOF decomposition using the forecast of the amplitudes. At each site, radial velocity forecasts are reconstructed as the sum of the mean field plus the first four EOF modes, multiplied by their time forecast amplitudes. Figures 5 and 6 show the forecast radial velocity fields at each radar site and for each of the analysed periods at 8-hour intervals over the first 32 hours of the forecast (right columns) together with the measured velocities (left columns). From 
TABLE I

NUMBER OF SSA EMPLOYED IN THE RECONSTRUCTION, VARIANCE EXPLAINED BY THESE MODES AND FORECASTING SKILL IN THE VALIDATION SET

\begin{tabular}{|c|c|c|c|c|c|}
\hline & \multirow[b]{2}{*}{$\mathrm{EOF}$} & \multicolumn{2}{|c|}{ First Period } & \multicolumn{2}{|c|}{ Second Period } \\
\hline & & SSA Variance $(\%)$ & Forecasting skill $(\%)$ & SSA Variance $(\%)$ & Forecasting skill (\%) \\
\hline \multirow{4}{*}{ PEY } & 1 & 87.6 & 98.8 & 95.1 & 97.3 \\
\hline & 2 & 88.3 & 98.8 & 90.6 & 91.8 \\
\hline & 3 & 91.4 & 99.6 & 86.3 & 88.1 \\
\hline & 4 & 92.0 & 89.7 & 88.9 & 89.6 \\
\hline \multirow{4}{*}{ POB } & 1 & 96.7 & 99.1 & 98.4 & 96.4 \\
\hline & 2 & 92.8 & 98.1 & 91.7 & 86.0 \\
\hline & 3 & 92.4 & 91.4 & 92.2 & 76.1 \\
\hline & 4 & 84 & 90.1 & 84.6 & 82.3 \\
\hline
\end{tabular}

these figures it can be observed that the forecasts do a reasonable job in capturing the radial velocity larger-scale spatial patterns and temporal variability.

The radial velocity forecast skill is quantified by a metric defined as

$$
<C_{X, \Re}>=\frac{\operatorname{COV}(X, \Re)}{\sigma_{X} \sigma_{\Re}}
$$

where $<>$ stands for the spatial mean, $X$ for the forecast/persistence velocities, $\Re$ for the measured velocity, $\mathrm{COV}$ for the covariance, and $\sigma$ for the standard deviation. The time evolution of this metric for both sites is displayed in Fig. 7, top panel for PEY station and bottom panel for POB station and for both periods. The proposed forecasting approach (squares) provides a significant improvement for the velocities when compared with the persistence at both sites (circles) and for the two periods. It is remarkable however that this method gives worst results, when compared with persistence, during the first 4 hours with a noticeable improvement afterwards in the velocity fields derived from the forecast. These results are slightly better for the radial velocities measured at PEY station indicating that some unpredictable signal, that can be related to noise in the measuring process deteriorates the forecast. 

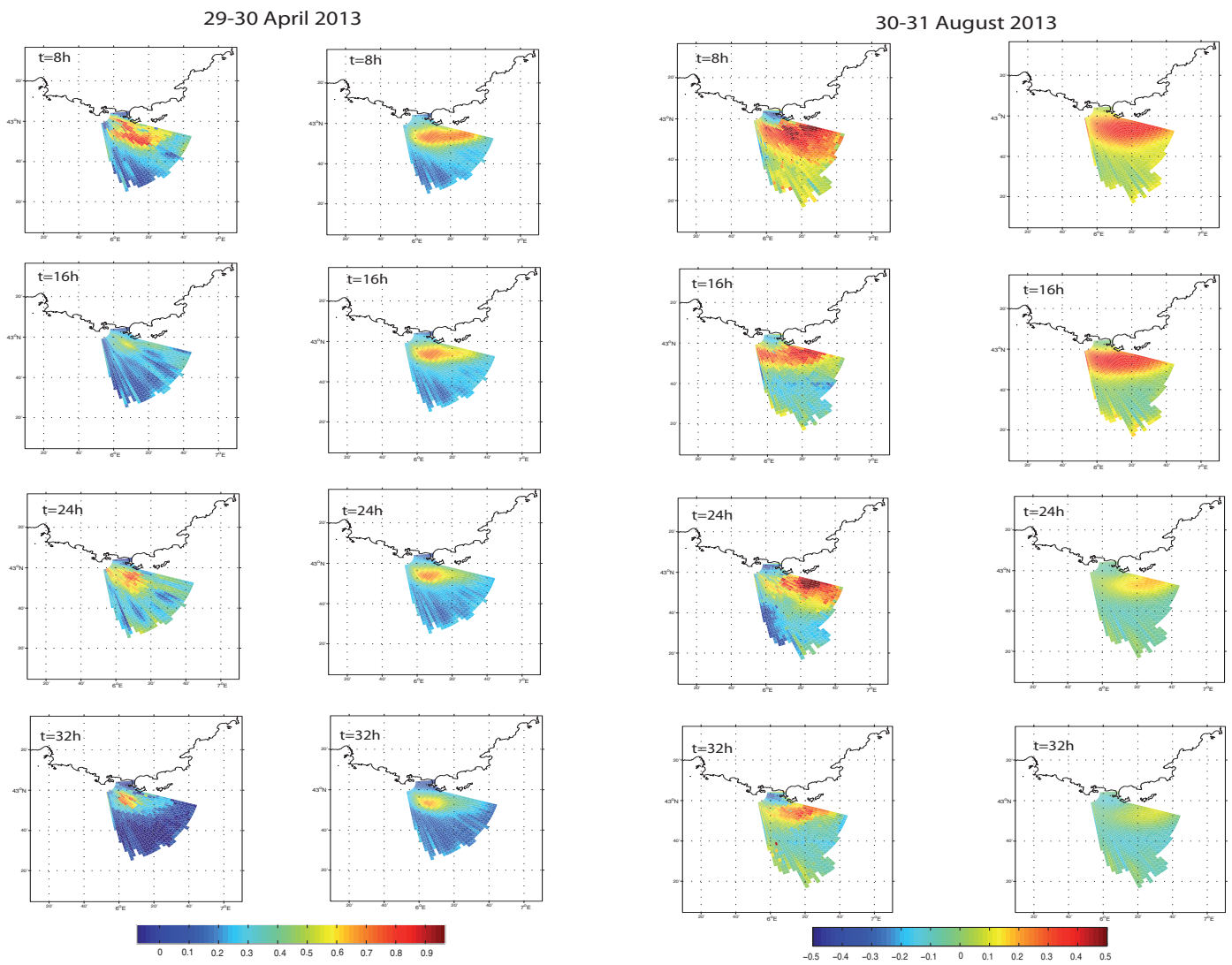

Fig. 5. Radial velocities (in m/s) at PEY station corresponding to April $29^{\text {th }}-30^{\text {th }}$ (left panel) and for August $30^{\text {th }}-31^{\text {st }} 2013$ (right panel). The first column of each panel shows the real (measured by the Hf-Radar) radial velocities while the second column of each panel shows the corresponding forecast. The forecast is made using the best GA equation obtained for each of the first four EOF used in the reconstruction. Snapshots are shown at 8 hour intervals over the first 32 hours of each of the forecast periods.

The performance of the proposed forecasting system is further analysed by comparing the spatially averaged difference between the forecast radial velocities and the measured ones for both periods. As shown in Fig. 8, the differences are for both PEY and POB antennas around $\pm 0.1 \mathrm{~m} / \mathrm{s}$ although the maximum and minimum difference obtained at any pixel (dashed lines) can be of $\pm 0.5 \mathrm{~m} / \mathrm{s}$. These large deviations are obtained mainly at the positions located in the border of the domain covered by each antenna.

In an operational forecasting system, total Cartesian velocities are obtained by combining the forecast radial velocities from the two sites. Fig. 9 display the vector difference between the reconstructed forecast velocities and the real ones at a 9 hours interval for both periods analyzed. The colors on each plot represent 


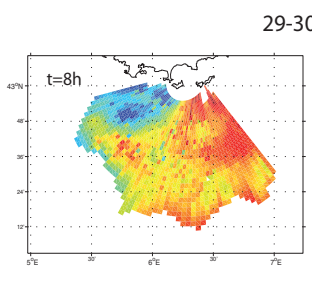

9-30 April 2013
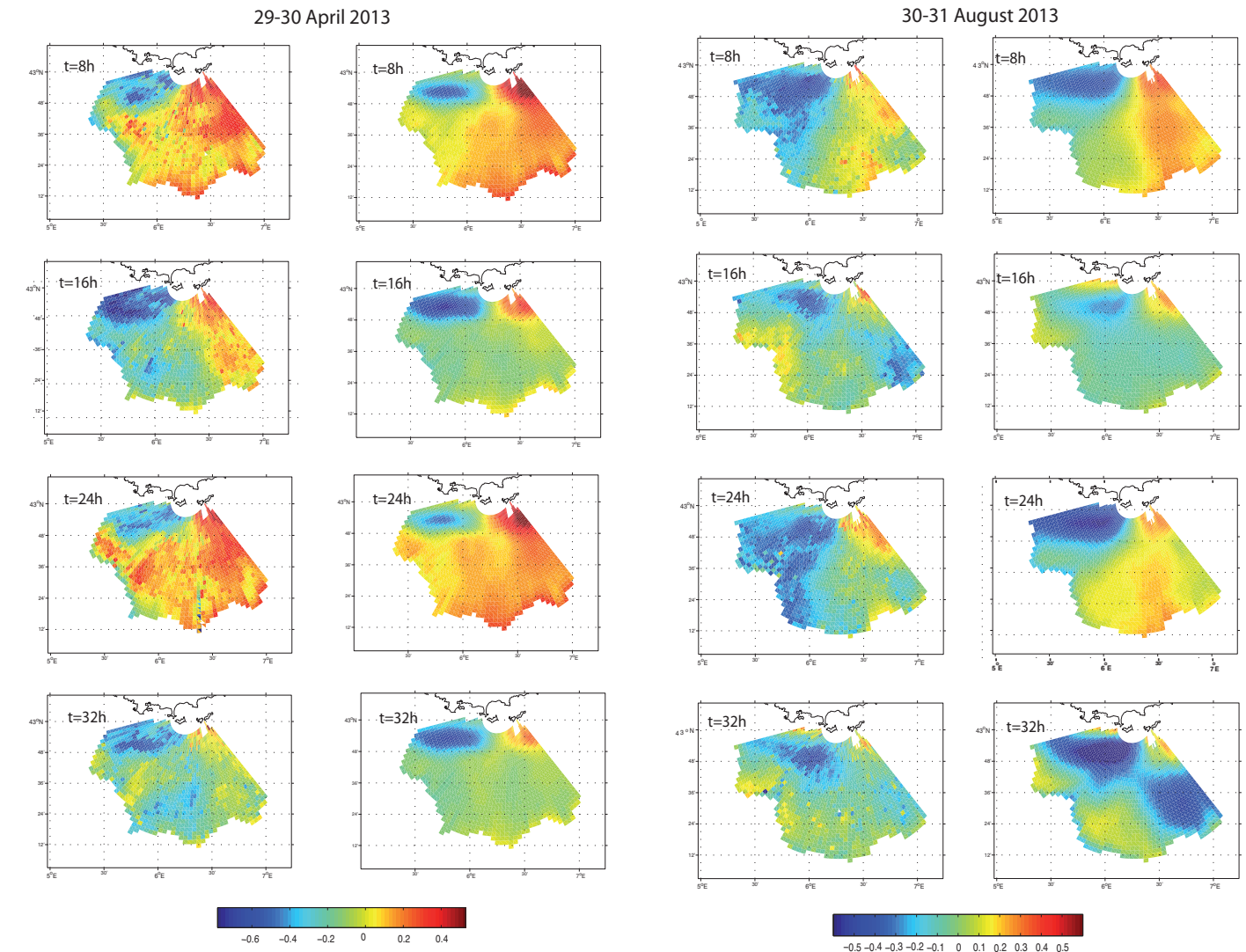

Fig. 6. Radial velocities (in $\mathrm{m} / \mathrm{s}$ ) at POB station corresponding to April $29^{\text {th }}-30^{\text {th }}$ (left panel) and for August $30^{\text {th }}-31^{\text {st }} 2013$ (right panel). The first column of each panel shows the real (measured by the Hf-Radar) radial velocities while the second column of each panel shows the corresponding forecast. The forecast is made using the best GA equation obtained for each of the first four EOF used in the reconstruction. Snapshots are shown at 8 hour intervals over the first 32 hours of each of the forecast periods.

the angular difference between both fields. As observed, the differences in the velocity magnitude between the forecast and the measurements are of the order of $|\vec{v}|=0.10 \mathrm{~m} / \mathrm{s}$ while the difference in the angle between both vectors can be of $\theta= \pm 30^{\circ}$.

To quantify the importance in the angular difference between both velocity fields the total velocity forecast skill is assessed using a time dependent Lagrangian metric (the Lagrangian distance, $\Delta$ ) that describes the evolving distance between particle pairs that are advected by two different velocity fields. This metric accounts for both spatial and temporal forecast errors. At each total velocity grid point, a massless particle is launched at the start of the forecast period, and passively advected by a velocity field. 

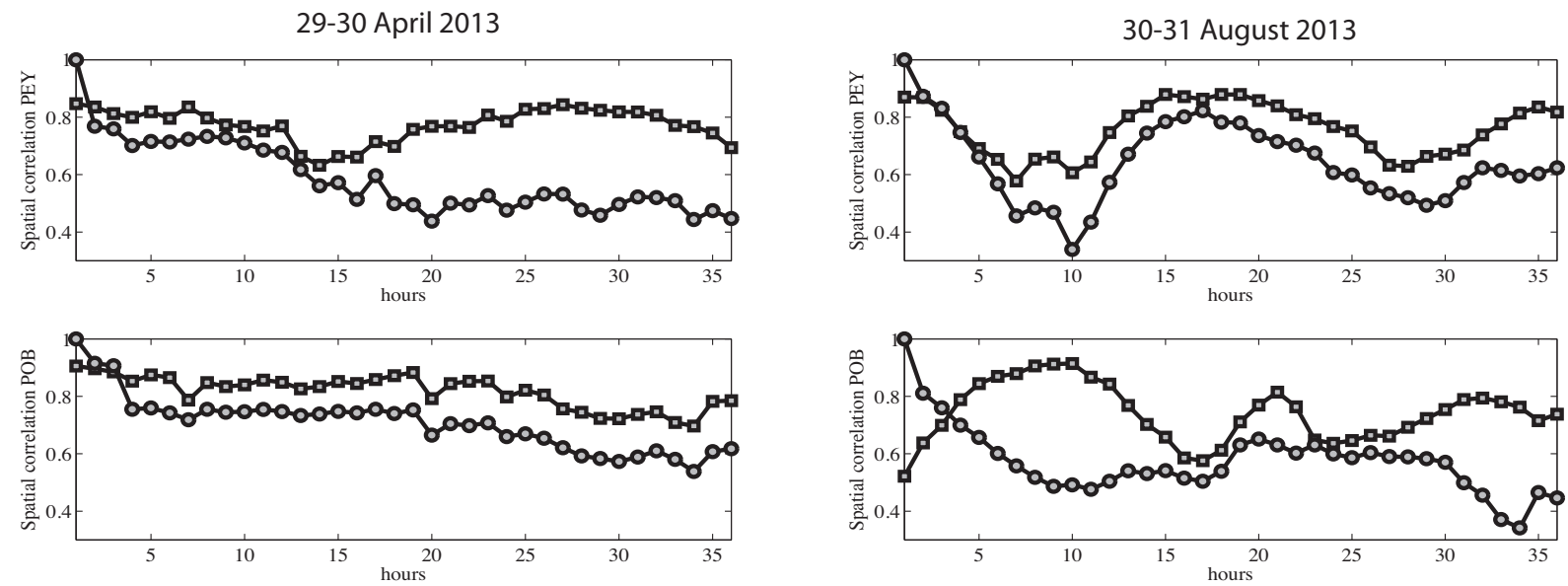

Fig. 7. Time series of spatially averaged correlation between instantaneous measured radial velocities and forecast radial velocities (squares) and between measured radial velocities and those from the persistence field (circles) at the PEY station (top) and POB station (bottom). The correlation is made for the 36 hour forecasting period. Left panels correspond for the forecast of April $29^{t h}-30^{t h}, 2013$ and the right panels for the forecast of August $30^{t h}-31^{\text {st }}, 2013$.
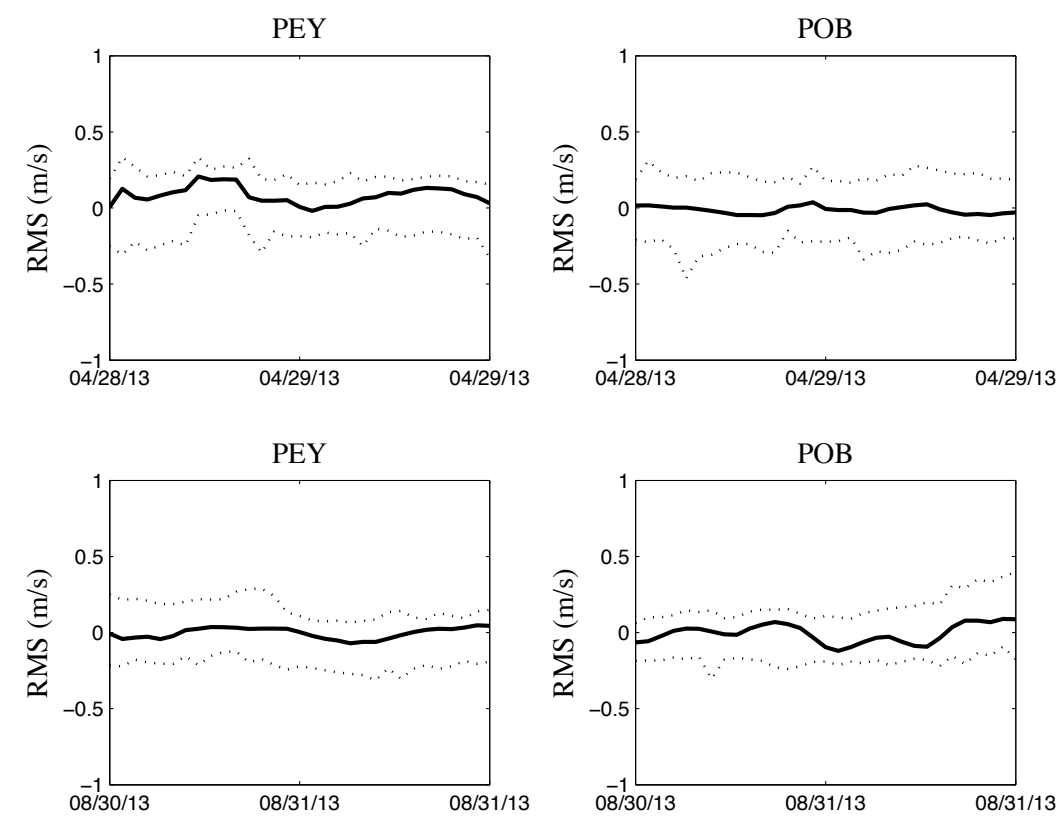

Fig. 8. Spatially averaged difference between forecast and measured radial velocities at PEY (left) and POB antenna (right) for the two forecasting periods. Dashed lines mark the maximum and minimum values found at any position for the difference.

Each particleś path is given by

$$
\mathbf{x}\left(t_{0}+\delta_{t}\right)=\mathbf{x}\left(t_{0}\right)+\int_{t_{0}}^{t_{0}+\delta t} \mathbf{u} d t
$$

Particle positions are determined at 30 minute intervals over the forecast period by numerically inte- 

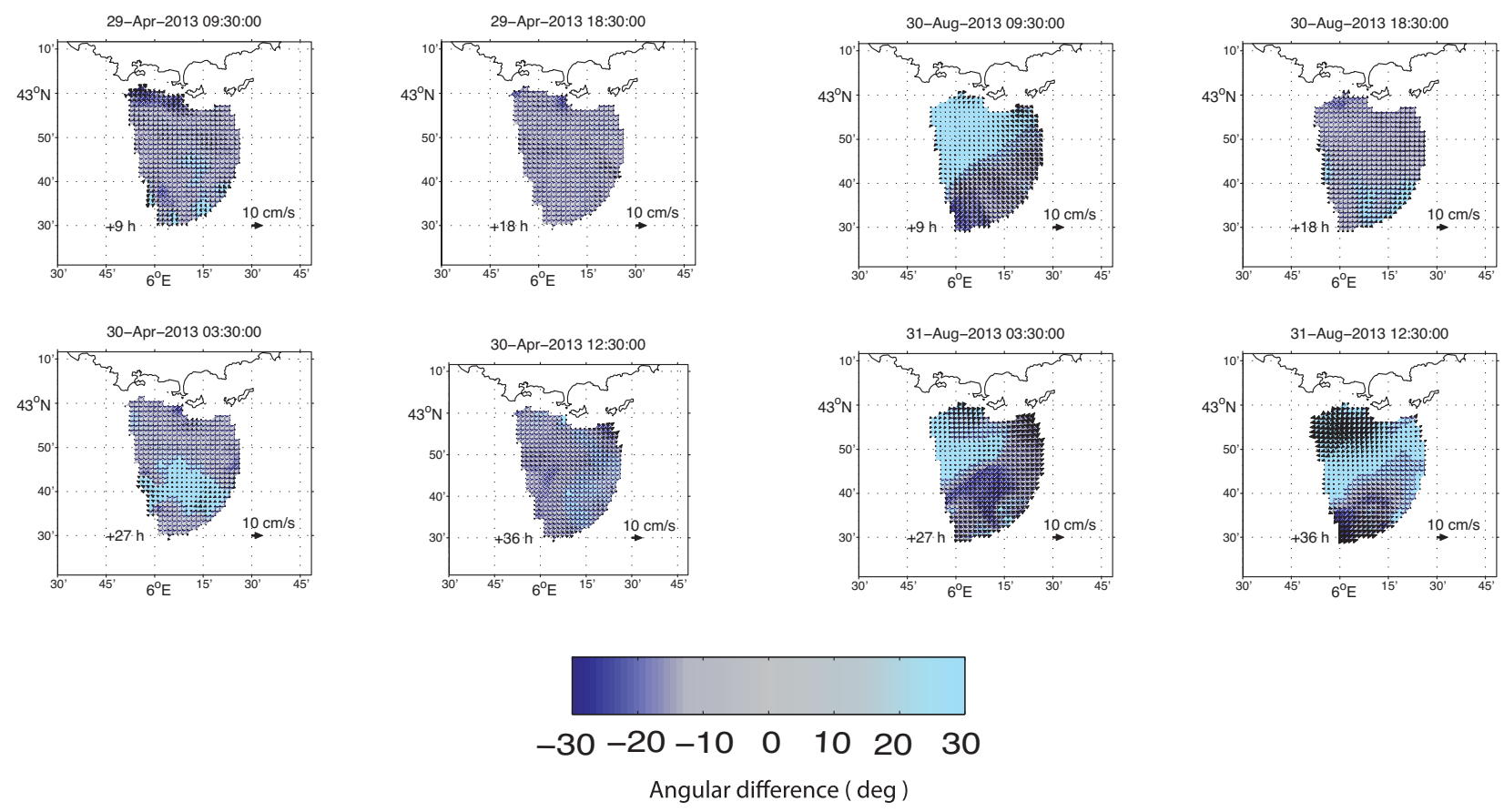

Fig. 9. Snapshots of vector difference between forecast velocities and measured ones for the forecast of April $29^{t h}-30^{t h} 2013$ (left) and for the forecast of Aug $30^{t h}-31^{\text {st }} 2013$ (right). The colors indicate the angular difference between both fields. Snapshots are provided at a 9 hours interval.

grating the Eulerian velocity in Eq. (5) with a fourth-order Runge-Kutta scheme. Particles that leave the domain are discarded from the analysis. For particle pairs, Lagrangian distance is computed as

$$
\Delta(t)=\sqrt{\left(x_{f}(t)-x_{r}(t)\right)^{2}+\left(y_{f}(t)-y_{r}(t)\right)^{2}},
$$

where the subscript $r$ denotes positions of particles advected with the measured velocity field and the subscript $f$ denotes positions of particles advected with a forecast or persistence velocity field. Here, $\Delta_{\text {forecast }}$ denotes $\Delta$ computed between observed and forecast velocity fields, and $\Delta_{\text {persistence }}$ denotes $\Delta$ computed between observed and persistence velocity fields.

Time series of $\Delta_{\text {forecast }}$ and $\Delta_{\text {persistence }}$ for the forecasting period of August $30^{t h}-31^{\text {st }} 2013$ are shown in Fig. 10. Snapshots are given at four hour intervals over the first 24 hours. As observed from Fig. 10 persistence velocities have better forecast skill over the first four hours of the forecast period, consistent with the time series of radial velocity forecast skill metrics shown in Fig. 7. The spatially averaged distance $\Delta$ for the persistence at $\mathrm{T}=4$ hours is of $\Delta_{\text {persistence }}=1500 \mathrm{~m}$ versus $\Delta_{\text {forecast }}=2500 \mathrm{~m}$. The GA was 
configured with a time delay of 1 (i.e. one hour so as to mimic the high frequency variability of the amplitudes (Fig. 3, (m)-(p) and 4, (m)-(p)) and therefore the mapping equations might introduce in the forecast some random variability at the short periods. However, for larger forecasting periods the error using the persistence increases notably. The spatially averaged difference at $\mathrm{T}=12$ hours is $9.1 \mathrm{~km}$ for the persistence field and $4.1 \mathrm{~km}$ for the predicted field and for $\mathrm{T}=24$ hours this error increases up to 14.5 $\mathrm{km}$ for the persistence and up to $5.5 \mathrm{~km}$ for the prediction with GA. Similar results for the Lagrangian metric are obtained for the winter period forecast.
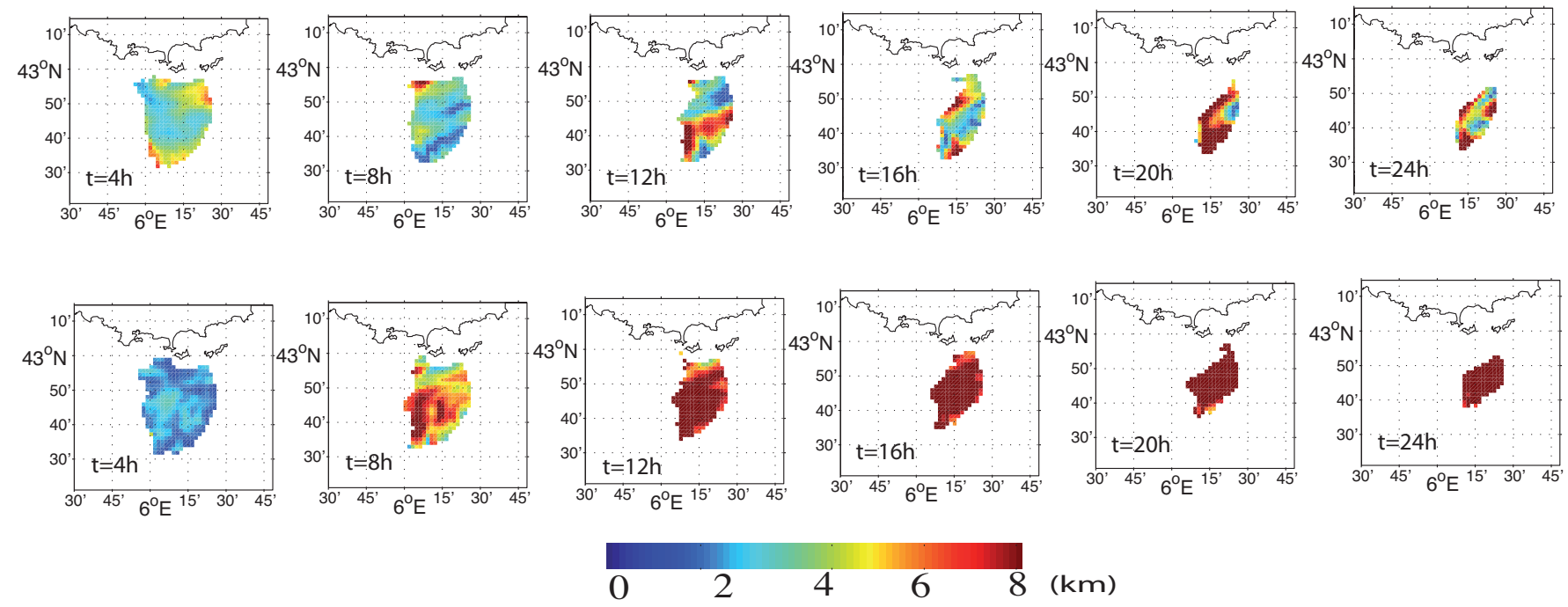

Fig. 10. Snapshots of $\Delta_{\text {forecast }}$ (top row) and $\Delta_{\text {persistence }}$ (bottom row ) at four hour intervals over the first 24 hours of the forecast period. Distances are in $\mathrm{km}$.

\section{CONCLUSIONS}

In the last years a large effort has been done worldwide in the design and construction of coastal observatories. These facilities obtain a large number of ocean variables that are routinely monitored and stored. Here we have presented an approach to obtain from HF-Radar measurements an empirical predictive model to forecast the coastal currents for a 36 hours period. The forecasting system has been applied and validated in the Var-Coastal area using a WERA system. To fill gaps in the radial velocities the DINEOF technique was applied over the original dataset. A good compromise for operational purposes 
is to recompute the DINEOF daily. Results indicate that, at least, part of the space-time ocean variability could be accurately forecast using radar data. More specifically, forecasts of hourly ocean surface currents are more accurate than those inferred by persistence fields after an horizon of 4 hours. We consider that for shorter times, the persistence of the HF-Radar system is a good alternative for the velocity fields. However, for longer periods, the use of the persistence ends in errors 2 times larger than those obtained by the presented model being the error 3 times larger after 24 hours.

The non-linear approach presented is very suitable for operational observing systems. The main advantages of forecasting directly the radial velocities is that first, no interpolation error is introduced before the forecast through the algorithm to obtain the Cartesian velocities and second, the forecast can be combined if more than three stations are available in coastal areas.

Future improvements of the method will imply to introduce the local wind at each station in a vectorial GA as well as in the acclimatization of the system in order to have in an operational way the forecasting of the currents with a delay of 4 hours.

\section{REFERENCES}

[1] Abramovich, Y. I., Johnson, B. A., Mestre, X., 2003. DoA estimation in the small-sample threshold region. In. Classical and Modern Direction-of-Arrival Estimation, Eds. T. E. Tuncer and B, Friedlander, Elsevier Academic Press, August 2009, Burlington, USA.

[2] Allou, A., Forget, P., Devenon, J.-L., 2010. Submesoscale vortex structures at the entrance of the Gulf of Lions in the Northwestern Mediterranean Sea.Continental Shelf Res. 30, 724-731.

[3] Alvarez, A., Orfila, A., Tintore, J., 2003. DARWIN: An evolutionary program for nonlinear modeling of chaotic time series. Comp. Phys. Comm. 136 (3), 334-349.

[4] Alvarez, A., Orfila, A., Sellschopp, J., 2003. Satellite based forecasting of sea surface temperature in the Tuscan Archipelago. Int. J. of Rem. Sensing 24 (11), 2237-2251.

[5] Alvarez, A., Orfila, A., Tintore, J., 2004. Forecasting the SST of the Ligurian Sea on weekly time scales with a satellite based ocean forecasting (SOFT) system. Journal of Geophysical Research 109 (C3).

[6] Alvera-Azcárate, A., Barth, A., Beckers, J.-M., Weisberg, R. H., 2007. Multivariate reconstruction of missing data in sea surface temperature, chlorophyll, and wind satellite fields. Journal of Geophysical Research: Oceans (1978-2012) 112 (C3).

[7] Alvera-Azcárate, A., Barth, A., Rixen, M., Beckers, J.-M., 2005. Reconstruction of incomplete oceanographic data sets using empirical orthogonal functions: application to the adriatic sea surface temperature. Ocean Modelling 9 (4), 325-346. 
[8] Alvera-Azcárate, A., Barth, A., Sirjacobs, D., Beckers, J.-M., 2009. Enhancing temporal correlations in eof expansions for the reconstruction of missing data using dineof. Ocean Science 5 (4), 475-485.

[9] Barrick, D. E., Evans, M.-W., Weber, B. L., 1977. Ocean surface currents mapped by radar. Science 198 (4313), $138-144$.

[10] Barrick, D. E., Fernandez, V., Ferrer, M. I., Whelan, C., Breivik, Ø. , 2012. A short-term predictive system for surface currents from a rapidly deployed coastal HF-Radar network. Ocean Dynamics 62 (5), 725-740.

[11] Barrick, D. E., Lipa, B. J., 1999. Using antenna patterns to improve the quality of seasonde HF-Radar surface current maps. Proc. of the IEEE Sixth Working Conference on Current Measurement, 5-8.

[12] Basu, S., Sarkar, A., Satheesan, K., Kishtawal, C. M., 2005. Predicting wave heights in the north indian ocean using genetic algorithm. Geophysical Research Letters 32 (17).

[13] Beckers, J. M., Rixen, M., 2003. Eof calculations and data filling from incomplete oceanographic datasets. Journal of Atmospheric and Oceanic Technology 20 (12), 1839-1856.

[14] Brankart, J., Brasseur, P., 1996. Optimal analysis of in situ data in the Western Mediterranean using statistics and cross validation. Journal of Atmospheric and Oceanic Technology 16, 477491.

[15] Canellas, B., Balle, S., Tintore, J., Orfila, A., 2010. Wave height prediction in the western mediterranean using genetic algorithms. Ocean Engineering 37, $742-748$.

[16] Elliot, A.-J., Jones, B., 2000. The need for operational forecasting during oil spill response. Marine Pollution Bulletin 40 (2), $110-121$.

[17] Emery, W.-J., Thomson, R.-E., 2004. Data Analysis Methods in Physical Oceanography. Elsevier H.V., Netherlands.

[18] Frolov, S., Paduan, J., Cook, M., Bellingham, J., 2012. Improved statistical prediction of surface currents based on historic HF-Radar observations. Ocean Dynamics 62 (7), 1111-1122.

[19] Guihou, K., Marmain, J., Ourmieres, Y., Molcard, A., Zakardjian, B., Forget, P., 2013. A case study of the mesoscale dynamics in the North-Western Mediterranean Sea: a combined datamodel approach. Ocean Dynamics 63 (7), 793-808.

[20] Gurgel, K.-W., Antonischki, G., Essen, H.-H., Schlick, T., 1999. Wellen radar (WERA): a new ground-wave HF-Radar for ocean remote sensing. Coastal Engineering 37 (3), 219-234.

[21] Lipa, B., Barrick, D., 1983. Least-squares methods for the extraction of surface currents from codar crossed-loop data: Application at arsloe. IEEE Journal of Oceanic Engineering, 8 (4), 226-253.

[22] Lipa, B., Nyden, B., Ullman, D. S., Terrill, E., 2006. SeaSonde Radial Velocities: Derivation and Internal Consistency. IEEE- Journal of Oceanic Engineering 31 (4), 850-861.

[23] Lorenz, E. N., 1965. A study of the predictability of a 28-variable atmospheric model. Tellus 17, 321-333.

[24] Molcard, A., Poulain, P., Forget, P., Griffa, A., Barbin, Y., Gaggelli, J., Maistre, J. D., Rixen, M., 2009. Comparison between $\{$ VHF $\}$ radar observations and data from drifter clusters in the gulf of la spezia (mediterranean sea). Journal of Marine Systems 78, Supplement (0), S79 - S89.

[25] Orfila, A., Alvarez, A., Tintore, J., Jordi, A., Basterretxea, G., 2005. Climate teleconnections at monthly time scales in the ligurian sea inferred from satellite data. Progress in Oceanography 66 (24), 157 - 170. 
[26] Orfila, A., Ballester, J. L., Oliver, R., Alvarez, A., Tintore, J., 2002. Forecasting the solar cycle with genetic algorithms. Astronomy and Astrophysics 386, 313-318.

[27] Preisendorfer, R. M., 1988. Principal Component Analysis in Meteorology and Oceanography. Developments in Atmospheric Science. Vol. 17. Elsevier, New York.

[28] Sayol, J.-M., Orfila, A., Simarro, G., Conti, D., Renault, L., Molcard, A., 2014. A Lagrangian model for tracking surface spills and SaR operations in the ocean. Environmental Modelling \& Software 52 (2), 74-82,

[29] Schmidt, R. O., 1986. Multiple emitter location and signal parameter estimation. IEEE Trans. Antennas and Propagation P-34, 276-280.

[30] Shay, L. K., Martinez-Pedraja, J., Cook, T. M., Haus, B. K., Weisberg, R. H., 2007. High-frequency radar mapping of surface currents using wera. Journal of Atmospheric and Oceanic Technology 24 (3), 484-503.

[31] Stewart, R.H., Joy, J.W., 1974. HF radio measurements of surface currents. Deep-Sea Res. 21, 1039-1049.

[32] Swinney, H. L., Gollub, J. P., 1986. Characterization of hydrodynamic strange attractors. Phys. D 18, 448-454.

[33] Takens, F., 1981. Detecting strange attractors in turbulence, in D. A. Rand and L.-S. Young. Dynamical Systems and Turbulence. Lecture Notes in Mathematics 898, 366-381.

[34] Tintoré, J., et al., 2012. SOCIB: The Balearic Islands Coastal Ocean Observing and Forecasting System Responding to Science, Technology and Society Needs. Marine Technology Society Journal, 47 (1), 101-117.

[35] Triantafyllou, G., Elsner, J. B., Lascaratos, A., Koutitas, C., A.Tsonis, A., 1995. Structure and properties of the attractor of a marine dynamical system. Mathematical and Computational Modelling 21, 73-86.

[36] Tsonis, A. A., Elsner, J. B., 1996. Mapping the channels of communication between the tropics and midlatitudes. Phys. D 92, 237-244.

[37] Vautard, R., Yiou, P., Ghil, M., 1992. Singular-spectrum analysis: A toolkit for short, noisy chaotic signals. Physica D: Nonlinear Phenomena 58 (14), $95-126$.

[38] Wunsch, C., 1996. The Ocean Circulation Inverse Problem. Cambridge University Press. 